\title{
A Near-infrared Survey of the Rosette Complex: Clues of Early Cluster Evolution
}

\author{
Carlos G. Román-Zúñiga ${ }^{1}$, Elizabeth A. Lada ${ }^{2}$ and Bruno Ferreira ${ }^{2}$ \\ ${ }^{1}$ Dept. of Astronomy, University of Florida, Gainesville 32611 \\ Present address: Harvard-Smithsonian Center for Astrophysics, Cambridge, MA USA \\ email: cromanzu@cfa.harvard.edu \\ ${ }^{2}$ Dept. of Astronomy, University of Florida, Gainesville 32611 \\ email: lada@astro.ufl.edu, ferreira@astro.ufl.edu
}

\begin{abstract}
The majority of stars in our galaxy are born in embedded clusters, which can be considered the fundamental units of star formation. We have recently surveyed the star forming content of the Rosette Complex using FLAMINGOS in order to investigate the properties of its embedded clusters. We discuss the results of our near-infrared imaging survey. In particular, we on the first evidence for the early evolution and expansion of the embedded clusters. In addition we present data suggesting a temporal sequence of cluster formation across the cloud and discuss the influence of the HII region on the star forming history of the Rosette.
\end{abstract}

Keywords. stars: early-type, stars: formation, infrared: stars, ISM: evolution, surveys, Galaxy: open clusters and associations

\section{Introduction}

Observational studies of embedded clusters are crucial to understand the early evolution of stellar systems. Embedded clusters are abundant in the many active Giant Molecular Clouds of the Milky Way, and tell us childhood stories that are common to the majority (60 to 90\%) of the stars in the galaxy (Lada et al. 1991; Carpenter 2000). Their observational properties place essential constraints for studies of larger and more evolved systems.

The Rosette Complex, located at $\mathrm{d}=1.6 \pm 0.2 \mathrm{kpc}$ in the constellation of Monoceros, is a valuable laboratory for the study of embedded clusters: it presents a convenient (and certainly impressive) layout of interaction between a HII region generated by a large OB association (NGC 2244) and a family of small clusters deeply embedded in an adjacent molecular cloud remnant. It has been suggested that the shock front generated by the HII region could have triggered the formation of the young clusters in the Rosette Molecular Cloud (Phelps \& Lada 1997, Williams, Blitz \& Stark 1995), following a process known as Sequential Star Formation (Elmegreen \& Lada (1977)). To test this hypothesis, it is crucial to investigate the influence of the local environment on the properties of the young clusters, as it would help us to understand the different initial conditions under which cluster formation occurs. Furthermore, studying the Rosette Complex allows to extend the embedded population surveys of nearby $(\mathrm{d}<500 \mathrm{pc})$ clouds beyond the local parsec neighborhood, something that until nowadays, has rarely been done.

As part of the NOAO survey program Toward a Complete Near-Infrared Spectroscopic and Imaging Survey of Giant Molecular Clouds (P.I.: Elizabeth A. Lada), we chose the Rosette Complex for a new systematic near-infrared study, focused on the investigation of its embedded cluster population (Román-Zúñiga et al. 2008.) Our main goals were to investigate the distribution of star formation in the Rosette, determine the fraction of 
stars that form in embedded clusters and compare these results to those found in other local clouds. We present here a brief summary of our study.

\section{Exploring the Rosette Complex}

One essential difficulty in our survey was the heavily populated background towards the Rosette Complex: at the anticenter of the Galaxy the field is composed of an abundant disk population and a tapestry of faint background galaxies; in the Near-infrared where much of the nebulosities of the cloud disappear, it is quite difficult to notice the cluster population against the sea of stars and galaxies. Our observations are sensitive to objects almost three times fainter than those observed with previous surveys like 2MASS, a clear advantage for the detection of young members, but unfortunately, at $1.6 \mathrm{kpc}$ it was a difficult task to separate galaxies, young low mass stars and objects with high color dispersion.

For those reasons we decided to apply (for this particular study) two major constraints to our survey: first, we limited our sample to a population of objects brighter than 15.75 (approximately $0.1 \mathrm{M}_{\odot}$ for unreddened Rosette stars) and presenting a near infrared excess (NIRX) in a JHK color-color diagram. For embedded stars, the color excess is a signature of circumstellar material and therefore of youth because it is known that $50 \%$ of the circumstellar disks in an embedded clusters will decrease in brightness by the second million year of age. Thus, the detection of infrared excess stars allows to highlight the most recent episode of formation in the star forming region. Second, we applied a technique of nearest neighbors specifically aimed to the detection of star clusters as regions with surface densities higher than the background calculated in a near control field (Ferreira \& Lada 2008, in preparation).

The combination of high surface density and circumstellar emission allowed us to confirm the existence of the seven embedded clusters identified visually by Phelps \& Lada (labeled PL01 to P107), and also indicated the existence of four more clusters, two heavily embedded in the molecular cloud (labeled REFL08 and REFL09), one hiding to the west of the OB association NGC 2244 (NGC 2237, as suggested by 2MASS data Li 2005) and a small cluster near a northwest faint clump (REFL10) at the Rosette Nebula region (see Fig. 1). The two smallest clusters, PL06 and RLEF10 have about 10 NIRX objects, indicating a total of around 30 members if the average JHK NIRX fraction in young clusters is close to $30 \%$. This is consistent with the minimum required for dynamical equilibrium (Adams \& Myers 2001; Lada \& Lada 2003), and let us think that we detected almost every active spot of formation in the cloud.

We analyzed the 11 Rosette clusters individually, and calculated their sizes, average extinction and NIRX fraction within the boundaries defined by contours of surface density. The properties of the clusters yield several interesting results: 1) the embedded clusters in the Rosette have an average JHK NIRX fraction around 30-40\%, which is consistent with those of clusters with ages of less than 2 Myr (e.g. slightly older than the Trapezium in Orion and younger then IC 348 in Perseus). This agrees with the spectroscopic age of $2 \pm 0.4$ Myr estimated for NGC 2244 (Park \& Sung 2002). 2) The average equivalent radii of the Rosette clusters are slightly larger than the average calculated by Lada \& Lada (2003) in a large sample of galactic embedded clusters, and the equivalent radii of individual clusters are negatively correlated with their mean extinction and NIRX fraction; this strongly suggests that the more evolved clusters are already less concentrated, and noticeably less embedded, all consistent with a picture of rapid infant evolution. In the Rosette, the embedded phase timescale would be similar to the T Tauri phase, as 


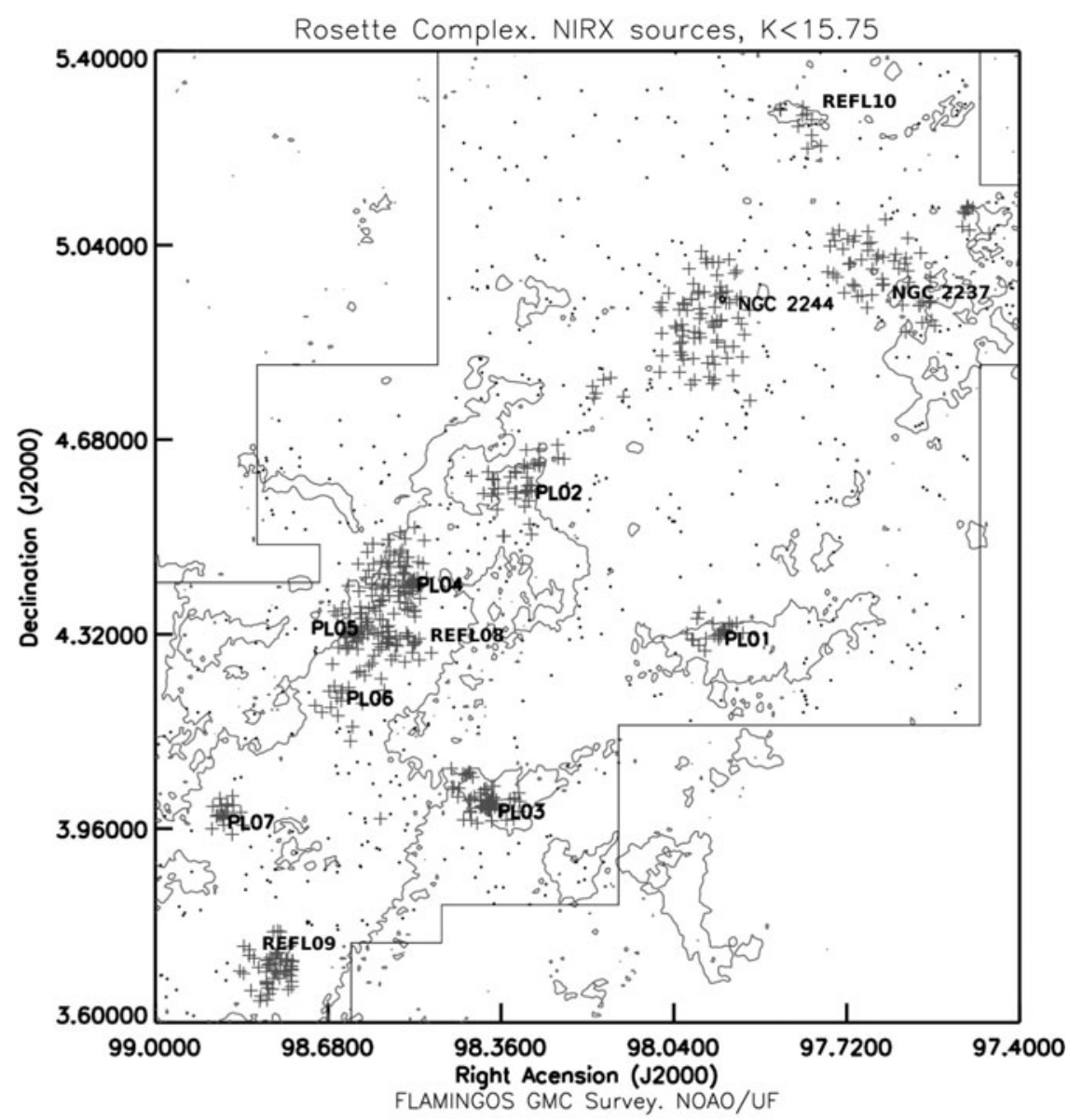

Figure 1. Identification of embedded clusters in the Rosette Complex. Both dot and plus symbols are sources identified as having near-infrared excess, but plus symbols indicate those objects with nearest neighbor surface densities above the background average, calculated in near control fields. The solid line contour indicate the base level of ${ }^{13} \mathrm{CO}$ emission of $0.8 \mathrm{~K} \cdot \mathrm{km} \cdot \mathrm{s}^{-1}$ that we used to define the extension of the main molecular cloud regions. The solid thin line indicates the limits of the NOAO survey coverage.

evidenced by the fact that a few stars in the more evolved embedded clusters are already visible in optical plates.

We found that $85 \%$ of the young stars in the Rosette Molecular Cloud belong to embedded clusters. This is in excellent agreement with previous studies in other clouds like Orion and Perseus (Lada et al. 1991, Carpenter 2000), confirming that the cluster mode of formation is highly dominant in galactic star forming complexes.

Finally, to investigate the influence of the HII region in the evolution of the star forming properties of the complex, we analyzed the distribution of young stars as a function of distance from the center of NGC 2244. We found that the radial distribution appear to show four major groups of clusters: the first group contains NGC 2244, NGC 2237 and RLEF10 in the Rosette Nebula, likely the older episode of formation in the cloud. The second group is located at the edge of the cloud that is in direct contact with the photodissociation region (the cloud 'Ridge', Blitz \& Thadeus 1980) and contains clusters PL01 and PL02; at the cloud ridge the molecular cloud is being compressed 
and destroyed systematically, possibly stopping the star formation process more quickly. The third group is located at the central core of the cloud, which is the region of main interaction between the nebula and the molecular cloud (the shock front), and contains clusters PL04, PL05, PL06, PL03 and REFL08, which account for almost 50\% of the total embedded population; at the central part of the cloud - where clusters formed from the most massive molecular gas clumps (Williams, Blitz \& Stark 1995) - the efficiency of formation could have been enhanced by the influence of the cloud. Finally, a fourth group of two clusters, PL07 and REFL09 is located at the back of the cloud, far away from the influence of the HII region. Were these lonely clusters formed spontaneously, without any influence from the Rosette Nebula?

We also found that if we calculate the average NIRX fraction for each of these groups, then this fraction appears to increase with distance from the nebula, indicating a possible sequence of cluster ages. The sequence of ages for embedded clusters in the Rosette Complex is really in agreement with the model of Elmegreen \& Lada (1977) because in that model, clusters form one after another, with one generation evolving and sweeping the material for the formation of the next one. In the Rosette all the clusters in the molecular cloud are still highly embedded and cannot be old enough to allow for a sequence of triggered formation. Instead, it appears that the clusters in the Rosette possibly have a sequence of ages that may be primordial and possibly reflects the early evolution of the molecular cloud. Then, the HII region affects the embedded population depending on how close is from each cluster. It will be necessary to constraint the relative ages of clusters using spectroscopy and mid-infrared photometry, to refine these interesting results. However, once we have a better idea of the real time scales involved in the formation of the Rosette Population we will be able to determine clearly its star forming history and to recollect what seems to be a very interesting story, one that could unlock many of the secrets of cluster formation.

\section{References}

Blitz, L. \& Thadeus, P. 1980, ApJ, 241, 676

Carpenter, J. M. 2000, AJ, 120, 3139

Elmegreen B. G. \& Lada, C. J. 1977, ApJ, 214, 725

Lada, E. A., DePoy, D. L., Evans, N. J., \& Gatley, I. 1991, ApJ, 371, 171

Lada, C. J. \& Lada, E.A. 2003, ARA\&A, 41, 57

Li, J. Z. 2005, ApJ, 625, 242

Park, B. \& Sung, H. 2002, AJ, 123, 892

Phelps, R. \& Lada, E. A. 1997, ApJ, 477, 176

Román-Zúñiga, C. G., Elston, R., Ferreira, B. \& Lada, E. A. 2008, ApJ, 695 (in press)

Williams, J. P., Blitz, L., \& Stark, A. A. 1995, ApJ, 451, 252 